\title{
Streiten im Chat
}

\author{
Martin Luginbühl (Zürich)
}

\begin{abstract}
While arguing in face-to-face communication is rather well investigated, there are almost no analyses on similar sequences of internet relay chats. Users of the IRC often revert to patterns of face-to-face communication, as it is for example shown in works on the phatic communication. But a lot of typical aspects of the modality "arguing" - as interruptions, overlaps, prosodic features etc. - can not be realized in the IRC. In exchange, this communication technology also creates new possibilities that are not available in face-to-face communication. In this article, I would like to ask what the consequences of these circumstances are for sequencies of arguing in the IRC. The main questions are:

- How is the appropriate modality of interaction created?

- What is the structure of sequencies of arguing? Which phases are identifiable (like cause, escalation, de-escalation)?

- Which blockades of interaction are realized?

- How is emotional involvement manifested?

Besides these questions concerning the form of sequencies of arguing, also the function of these sequencies shall be analyzed. This question is important, because arguing in the IRC always is arguing in front of an audience and because the possible anonymity can have an influence on their function.
\end{abstract}

\section{$1 \quad$ Fragestellung}

Ausgangspunkt meiner Untersuchungen zum Thema Streiten im Chat ist die Frage, wie Kommunikationsteilnehmer/-innen unter den medialen Bedingungen des Chats die kommunikative Handlung Streiten bewältigen. Diese Frage interessiert mich im Hinblick auf verschiedene Aspekte:

- Die Frage ist "gesprächs"analytisch interessant, weil viele Merkmale von Streit, wie sie von der linguistische Gesprächsanalyse eruiert wurden und ich sie noch kurz erläutern werde, im Chat nicht realisierbar sind (z.B. Unterbrechungen ${ }^{1}$ oder Überlappungen, aber auch bestimmte Ausdrucksweisen auf para- und nonverbaler Ebene). Die Kommunikationsteilnehmer/-innen im Chat können hier also auf bestimmte, zentrale

\footnotetext{
${ }^{1}$ Unterbrechungen sind im moderierten Chat möglich (s. dazu Storrer 2001b), Gegenstand der vorliegenden Untersuchung sind aber ausschließlich unmoderierte Chats.
} 
Muster der gesprochenen Kommunikation nicht zurückgreifen. Welche Konsequenzen hat dies für Streitsequenzen?

- Ebenfalls von Interesse ist die Frage, in welchen Chats worüber gestritten wird und wie häufig dies vorkommt. Die vorliegende Analyse erlaubt keine repräsentativen Aussagen, gibt aber dennoch Hinweise zur Beantwortung dieser Frage.

- Zudem ist bei einigen Streitsequenzen, bei denen z.B. jemand Beleidigungen und Beschimpfungen realisiert, auch die Frage nach der sozialen Kontrolle im Chat tangiert. Wie reagieren Chatter/-innen auf derartige Beiträge und wie gelingt es ihnen, unsoziales Verhalten zu unterbinden? (Vgl. dazu auch Döring 2001.)

\section{$2 \quad$ Zum Terminus Streit}

Unter Streit verstehe ich nach Carmen Spiegel (1995: 17) eine verbale, kontroverse und unkooperative Austragungsform von Konflikt, die unter anderem durch Missachtung des Partnerimages gekennzeichnet ist. Zentral sind einerseits die Unkooperativität und andererseits die Verletzungen des Partnerimages. Ohne diese Merkmale würde man eher von einer dissenten Sequenz im Sinne von Gruber (1996) sprechen, in welcher konträre Standpunkte formuliert und anschließend ausgehandelt werden. Beim Streiten aber gerät der Beziehungsaspekt in den Vordergrund, wodurch das Verlieren bedrohlicher wird, weil im Streit die Identitäten ausgehandelt werden. Deshalb eskalieren derartige Kommunikationsereignisse auch rasch, weil bei einer Identitätsbedrohung die Emotionen intensiver werden. Die Positionen werden dann oft explizit und personenbezogen gestaltet (z.B. Vorwurf, Kritik, Beleidigung etc.), ohne dass die eigene Position weiter entfaltet werden kann. Neben derartigen eher inhaltlichen Aspekten zieht Streit - wie andere dissente Sequenzen - auch eine bestimmte Gesprächsorganisation nach sich, welche von Gruber Dissensorganisation genannt wird (Gruber 1996: 56). Diese ist etwa charakterisiert durch unkooperative Sprecherwechsel (DRP, disagreement relvant places, ebd.: 60), eine dissensorientierte Präferenzorganisation und mehr formale Kohäsion. Tritt nun im Streiten eine derartige Dissensorganisation gleichzeitig mit Emotionen auf, so droht die Kommunikation zusammenzubrechen.

Streitsequenzen in mündlichen Gesprächen lassen sich in vier Phasen einteilen: Anlass, Eskalation, Beendigung und Nachverbrennung (dazu Gruber 1996 und Spiegel 1995).

Den Anlass bildet ein Verstoß gegen den Gesprächskonsens, z.B. bezüglich der Ansichten über die Welt, die Rollenverteilung in der Kommunikation etc. Meist handelt es sich hier um Widersprüche und Vorwürfe (Gruber 1996: 83-90).

Wird dieser Anlass von anderen Teilnehmern aufgegriffen und moniert, so liegt der Beginn einer dissenten Sequenz vor und es kann zu einer Eskalation und somit zum Streit kommen. Diese weist dann eine entsprechende Dissensorganisation auf (mit lautem Sprechen, Unterbrechungen etc.) und wird emotional geführt, indem z.B. mit negativ bewertenden Lexemen auf die Beziehungsebene übergegriffen wird. 
Eine Streitsequenz kann unterschiedlich beendet werden: Das Problem und somit der Konflikt können gelöst werden, das Thema und die Interaktionsmodalität können gewechselt werden, ohne den Konflikt zu bearbeiten, oder die Kommunikation kann abgebrochen werden. Deeskalierend wirken oft ruhiges Sprechen und Freundlichkeiten verschiedener Art (Spiegel 1995: 24-26).

Unter Nachverbrennungen wird die Thematisierung von Imageverletzungen im Anschluss an den Hauptgesprächsstrang verstanden.

\section{$3 \quad$ Korpus}

Das hier untersuchte Korpus stammt aus dem "bluewin-chat"2. Der "bluewin-chat" ist nicht nur der größte Chat der Schweiz, sondern auch einer der größten Chats im deutschsprachigen Raum. In den Kanälen des "bluewin-chat" lagen die Teilnehmerspitzen im Oktober 2002 durchschnittlich bei etwa 3800 Personen, im Janaur 2003 bei etwa $3200 .^{3}$

Das Korpus basiert auf Chat-Mitschnitten aus der Zeit vom 20. August 2002 bis zum 28. Oktober 2002; insgesamt wurden gut 27 Stunden Chat auf diversen Channels des "bluewinchat" aufgezeichnet und analysiert. Die Aufzeichnungen wurden an 4 Tagen gemacht, und zwar jeweils zeitgleich in 4 bis 5 Channels. Aufgezeichnet wurde immer der thematisch nicht spezifizierte "Bluewin"-Channel, in dem sich immer sehr viele Chatter aufhielten. Zusätzlich wurden - mit Ausnahme des ersten Aufzeichnungstages - jeweils noch 3 bis 4 thematisch spezifizierte Channels aufgezeichnet. Dabei wurden Channels gewählt, in denen jeweils mehr als 20 Chatter/-innen eingeloggt waren. Dies waren: "Eishockey", "Fußball", "HipHop", "Metal" und in einem Fall "Punk". Die Datenerhebung erfolgte nonreaktiv mittels Verhaltensbeobachtung.

Flirt-Channels habe ich nicht in die Analyse mit einbezogen, da ich hier aufgrund erster, allerdings nicht systematischer Beobachtungen keine Streitsequenzen beobachtet habe. Allerdings müsste man diese Channels bei einer weiteren Studie unbedingt mitberücksichtigen, da sich hier auch Konflikte ergeben, z.B. im Hinblick auf die Explizitheit der Formulierung sexueller Wünsche, aber auch im Hinblick auf Belästigungen etc. (dazu auch Dörig 2001). Ebenfalls unberücksichtigt blieben fremdsprachige Channels.

Insgesamt ergab sich so ein Korpus von gut 260 Seiten Chatprotokollen.

\section{$4 \quad$ Analyse}

\subsection{Häufigkeit der Streitsequenzen und Streitgegenstand}

Probleme - und dies ist eigentlich bereits ein erster Befund - ergaben sich hinsichtlich der Auffindung von Sequenzen, in denen gestritten wird. In der Sekundärliteratur wird zwar teilweise gesagt, man finde "ohne langes Suchen" "schwer erträgliche Beschimpfungen und Beleidigungen" in öffentlichen Chats (Döring 2001: 111). Im Schweizer "bluewin-chat" kann

\footnotetext{
2 http://www.bluewin.ch/chat.

3 Angaben von http://www.webchat.de; abgefragt am 1. November 2002 und am 1. Februar 2003.
} 
davon aber nicht die Rede sein. Ich habe in den analysierten Protokollen insgesamt 14 Sequenzen gefunden, welche Merkmale von Streitsequenzen aufweisen. Dabei handelt es sich um Sequenzen unterschiedlicher Länge. Sie umfassen manchmal nur 7 Äußerungen, in einem anderen Fällen über 100 .

Natürlich bezieht sich diese Zahl nur auf Streitsequenzen, die 'öffentlich', also nicht in Privaträumen zu beobachten waren. Vielleicht hat diese niedrige Fallzahl damit zu tun, dass eher in Privaträumen gestritten wird und nicht an der Öffentlichkeit. Ein Indiz hierfür ist die Tatsache, dass im thematisch nicht spezifizierten, aber meist sehr gut frequentierten "bluewinchat" keine einzige längere Streitsequenz vorliegt. Diese längeren Sequenzen finden sich alle in kleineren, also 'privateren' Channels wie eben "Eishockey" oder "HipHop".

Worüber wird in den einzelnen Channels gestritten? Im Channel "bluewinchat" wird, wie erwähnt, kaum öffentlich gestritten. Zwar wird wiederholt von einigen Chattern beklagt, dass es in diesem Channel langweilig sei, was als indirekter Angriff interpretiert werden kann, aber meist ohne Reaktionen bleibt. Konflikte können in allen anderen Channels ausgelöst werden durch das Verhalten einzelner Chatter/-innen - wenn einzelne z.B. die anderen Chatter/-innen kollektiv beleidigen. In einem Fall führte auch die Wahl eines Nicks zu einer kurzen Streitsequenz ${ }^{4}$. Einen weiteren - erwartbaren - Streitanlass bilden bewertende Äußerungen einzelner Eishockeyclubs, Fußballclubs oder Musikstilrichtungen in den entsprechenden Channels. In je einem Fall leiteten Äußerungen über die Herkunft eines Chatters bzw. eine Äußerung über Ausländer eine Streitsequenz ein (beides im Channel "Eishockey").

In der Chatiquette der bluewin-Channels wird das Streiten direkt thematisiert. So heißt es etwa unter dem Stichwort "Respekt" in einer Positivregel:

Denk daran: Am anderen Ende der Leitung sitzt auch ein Mensch, keine Maschine! Geh darum mit jedem so um, dass Du ihr/ihm auch im wirklichen Leben noch in die Augen sehen kannst. Also so, wie Du selber gerne behandelt werden würdest. Zeige Respekt und dränge dich niemandem auf. Wenn dich jemand nicht respektiert, dann versuche einfach, ihn zu ignorieren, anstatt dich mit ihm zu streiten. ${ }^{5}$

$\mathrm{Ob}$ in den Channels so wenig gestritten wird, weil sich die meisten an diese Regel halten, kann ich nicht beurteilen. Andere Regeln - etwa den Rat, die Frage "Will hier jemand mit mir chatten?" zu unterlassen - werden auf jeden Fall nicht befolgt. Interessant an der obigen Regel ist auf jeden Fall der Tipp, jemanden, der einen nicht respektiert, zu ignorieren. Vorgeschlagen wird eine negative Sanktion - wobei offen bleibt, ob hier eine soziale Sanktion gemeint ist (sich abwenden) oder eine technische (Ignore-Befehl).

\subsection{Streitanlass und Reaktionen}

Bei der Durchsicht meines Materials fällt zunächst einmal auf, dass es in der Tat eine ganze Reihe von Äußerungen gibt, die als Streitanlass geeignet wären, auf die aber nicht öffentlich reagiert wird.

\footnotetext{
${ }^{4}$ Ein Chatter provozierte im Channel "Metal" am 3.10.02 den Chatter "leamas" folgendermaßen: "hm namen rückwärts schreiben ist schon was tolles und sowas von originell... *gääähn*", worauf der angesprochene Chatter u. a. mit folgender Äußerung reagiert: "u du ufem aafiggitrip?" (Übersetzung: "und du auf dem anficktrip?").

5 http://www2.bluewin.ch/chat/services/chatiquette d.html; abgefragt am 1. Februar 2003.
} 
Zwei typische Beispiele sollen dies illustrieren:

gatto: isch ja mega langwilig do! (Übersetzung: "Ist ja mega langweilig

hier") (Channel "Bluewin" 20. 10. 02)

SCB-Star: H C D S T I R B (Channel "Eishockey" 3. 10. 02)

Beide Äußerungen blieben ohne öffentliche Reaktionen im Chat. Im Vergleich zu mündlichen Kommunikationssituationen ist dies doch recht erstaunlich. Würden SCB und HCD-Fans an einem Tisch sitzen und jemand würde die eben zitierte Äußerung realisieren, ist es kaum vorstellbar, dass darauf nicht reagiert wird. Allerdings ist das Nicht-Reagieren im Chat einfacher als in einer face-to-face-Situation. Aufgrund der Anonymität ist die Verpflichtung, kommunikative Verantwortung zu übernehmen - also z. B. relevante Äußerungen zu realisieren - kleiner. Zudem ist aufgrund des "Mühlen-Prinzips" (Wichter 1991: 78-79) ignorieren einfacher, da der Status einer (auf dem Bildschirm) nachfolgenden Äußerung nicht offensichtlich ist; es kann sich um eine bewusste Nicht-Reaktion handeln oder um eine Äußerung, die nur aus technischen Gründen erst nach der ersten Äußerung auf dem Bildschirm erscheint, aber eigentlich vor der ersten Äußerung abgeschickt wurde.

Wie viele private Reaktionen dadurch hervorgerufen wurden, kann nicht eruriert werden. Allerdings weist ein Feldexperiment von Döring (2001), in dem öffentlich nach sexuellem Kontakt gesucht wurde, darauf hin, dass Reaktionen mit negativen sozialen Sanktionen überwiegend öffentlich realisiert werden. In Dörings Korpus waren über 70\% der Reaktionen auf ihre Äußerung öffentlich.

Dieses Fehlen von öffentlichen Reaktionen ist typisch für Äußerungen, die als Beleidigungen oder Angriffe interpretiert werden können, aber nicht direkt an jemanden gerichtet sind. Indirekte Angriffe führen in meinem Korpus nur dann zu Streitsequenzen, wenn sie wiederholt geäußert werden. Auf direkte Angriffe wird hingegen in der Regel reagiert. Das folgende Beispiel illustriert dies (Channel "Eishockey", 28. 10. 02; Auslassungen in eckigen Klammern []):

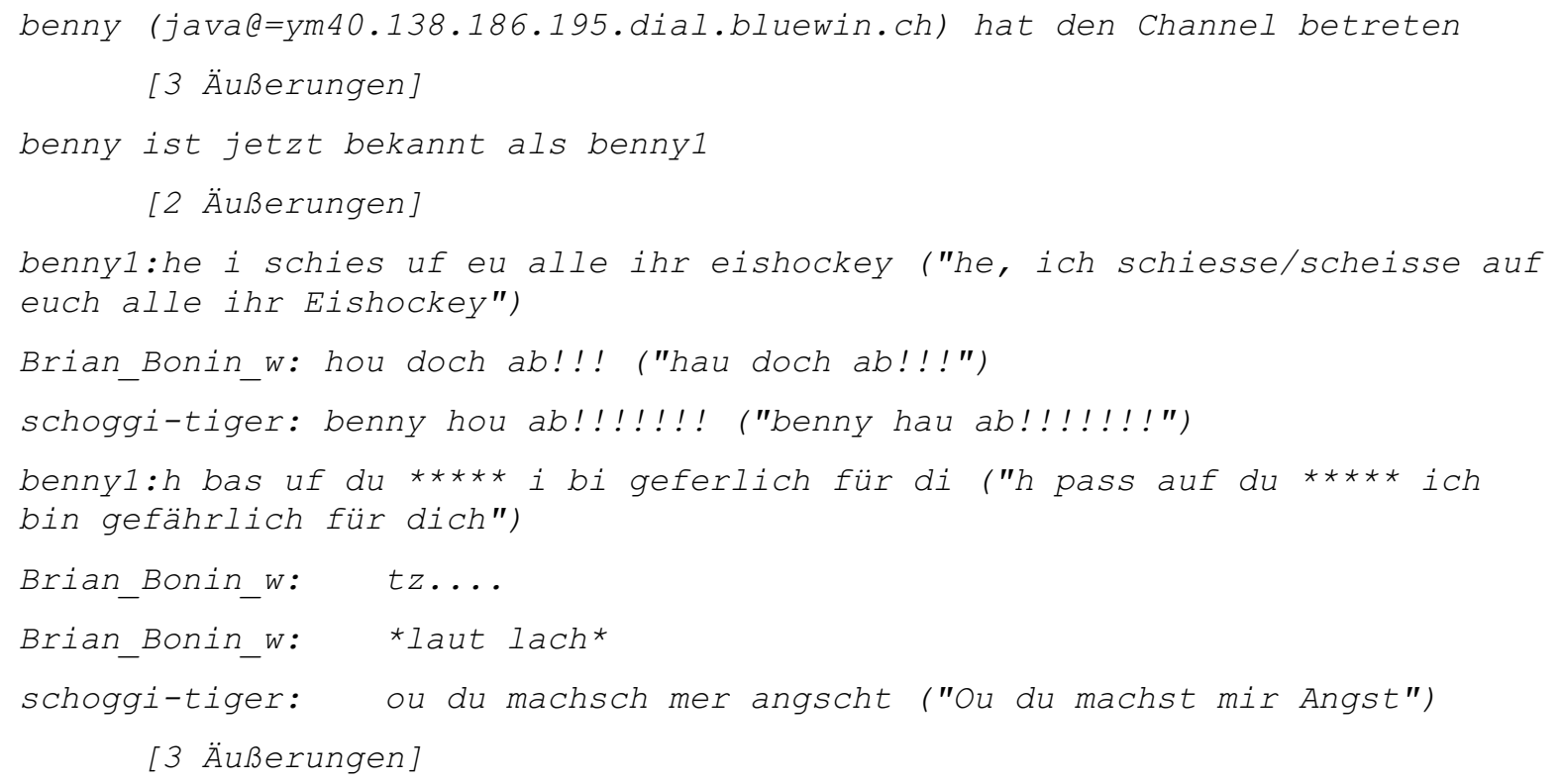




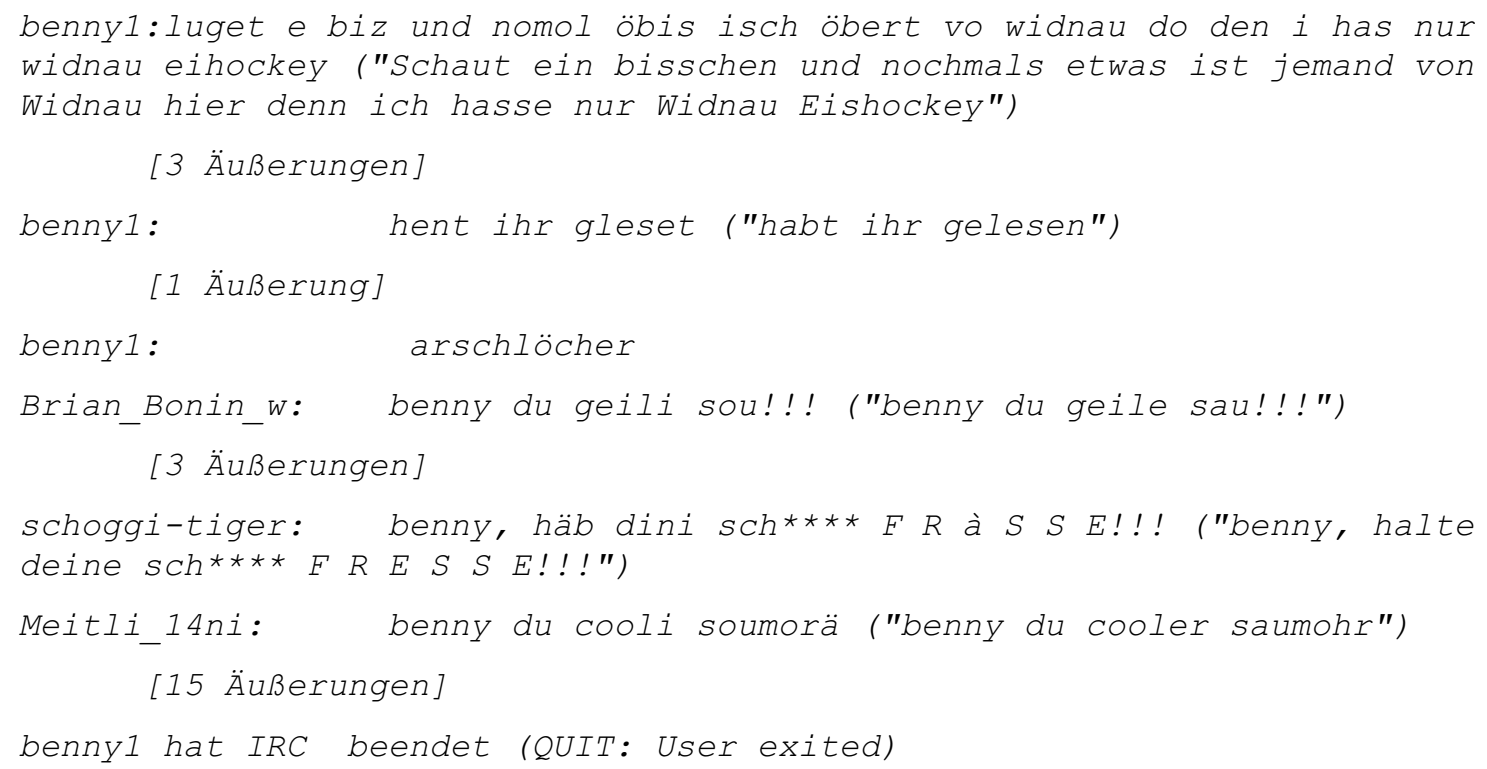

"Benny1" gibt gleich in seiner ersten Äußerung seine Verachtung für die Eishockey-Fans zum Ausdruck, und zwar mit einer stark bewertenden Formulierung - wobei infolge des Dialektgebrauchs unklar bleibt, ob mit "schies" das Verb "schießen" oder "scheißen" gemeint ist. Mit dieser Formulierung werden die anwesenden Chatter/-innen direkt angesprochen. Diese beleidigende Äußerung evoziert starke Reaktionen von verschiedenen Chattern, die ebenfalls auf der Beziehungsebene einzuordnen sind und in vier Fällen durch Satzzeichenreduplikation Prosodie emulieren und so Emotionalität anzeigen. ${ }^{6}$ Unklar bleibt die Bedeutung der Gefühlsäußerung *laut lach*. Diese Äußerung von "Brian_Bonin_w" kann als Auslachen interpretiert werden (Da kann ich nur laut lachen), aber auch eine Wendung ins Humoristisch-Spielerische anzeigen und könnte so eine Deeskalation einleiten. Darauf würde die nächste, in ihrer Bedeutung ebenfalls ambivalente Äußerung von "Brian_Bonin_w" hinweisen ("benny du geili sou!!!" "benny du geile sau!!!"). Überhaupt sind im Chat viele derbe Schlagabtausche mit Signalen durchsetzt, welche dazu dienen eine unernste Interaktionsmodalität abzusichern. Ich komme auf diesen Aspekt später noch zurück.

Insgesamt aber hat "Benny1" mit seiner Äußerung überwiegend klar negative, emotionale Reaktionen evoziert. Auffallend ist, dass "Benny1" von anderen Chattern aus dem Channel verwiesen wird (ein derartiger Verweis entspricht in der Terminologie von Döring einer öffentlichen, sozialen Sanktionsmethode; Döring 2001: 125, 132) oder dass mit Spott und Beleidigungen reagiert wird.

Neben diesen negativen Sanktionen der angegriffenen Chatter/-innen gibt es auch den Fall, wo ähnliche Äußerungen einen technischen Channel-Verweis durch den Operator zur Folge haben. Ich habe zwei Beispiele in einem Channel gefunden, wo ein Chatter bereits bei nicht direkt an andere Chatter/-innen formulierte potenzielle Angriffe aus dem Channel gekickt wird (beides: Channel "Fußball", 28.10.02):

\footnotetext{
${ }^{6}$ Die Satzzeichenreduplikation könnte ein Ersatz für Lautstärke sein, dazu Haase et al. (1997).
} 
EHCBIEL: Bayern raus!!!!!

[2 $\ddot{\text { ÄuBerungen] }}$

ChanServ hat Ewok[LaCoruna-0-0-Bayern] geoppt

[1 Äußerung]

EHCBIEL wurde aus dem Channel von Ewok[LaCoruna-0-0-Bayern] geworfen (du au) (Übersetzung: "(du auch)")

Italygirl: bayern?hahahahah

[6 ÄuBerungen]

ChanServ hat Ewok[LaCoruna-0-0-Bayern] geoppt

Italygirl wurde aus dem Channel von Ewok[LaCoruna-0-0-Bayern] geworfen

(bye! bitte nix gägä bayern! thx) ("(bye! bitte nichts gegen bayern! thx)")

ChanServ hat Ewok[LaCoruna-0-0-Bayern] entoppt

ChanServ hat Ewok[LaCoruna-0-0-Bayern] geoppt

EHCBIEL wurde aus dem Channel von Ewok[LaCoruna-0-0-Bayern] geworfen (du

dörfsch grad dusä bliebä!) ("(du darfst gleich draußen bleiben!)")

Ewok[LaCoruna-0-0-Bayern] hat "EHCBIEL! *java@=OSuxbe-99-357-309-

776.adslplus.ch" vom Channel verbannt

In diesem Channel bzw. bei diesem Operator herrscht offensichtlich eine andere Norm als in den anderen von mir beobachteten Channels. Neben diesen zwei Sequenzen habe ich in diesem Channel keine Streitsequenzen oder streitähnlichen Sequenzen gefunden. Ob und wie gestritten wird, hängt somit stark von den Normen des jeweiligen Channels ab.

Beim Beispiel mit "Benny1" habe ich darauf hingewiesen, dass sich die Chatter/-innen in Streitsequenzen sehr rasch direkt angreifen, sich direkt bewerten und beleidigen. Diese Beobachtung, dass in Streitsequenzen ohne lange Vorlaufszeit sehr rasch direkt den Chatpartner bzw. die -partnerin bewertende und beleidigende Äußerungen realisiert werden, ist typisch für die Streitsequenzen, die durch direkte Angriffe veranlasst werden.

Zwei Beispiele mögen dies illustrieren. Das erste stammt aus dem Channel "Punk" vom 3.10.02:

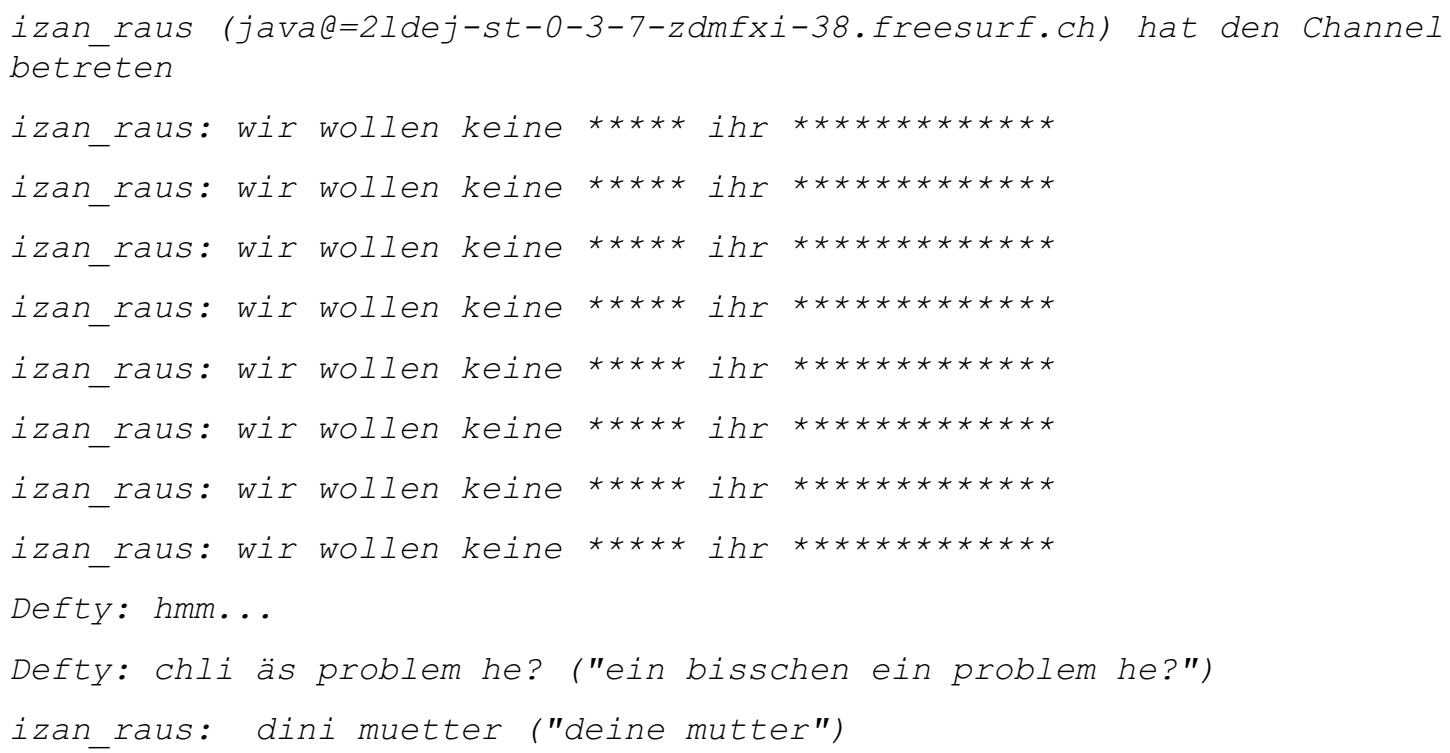




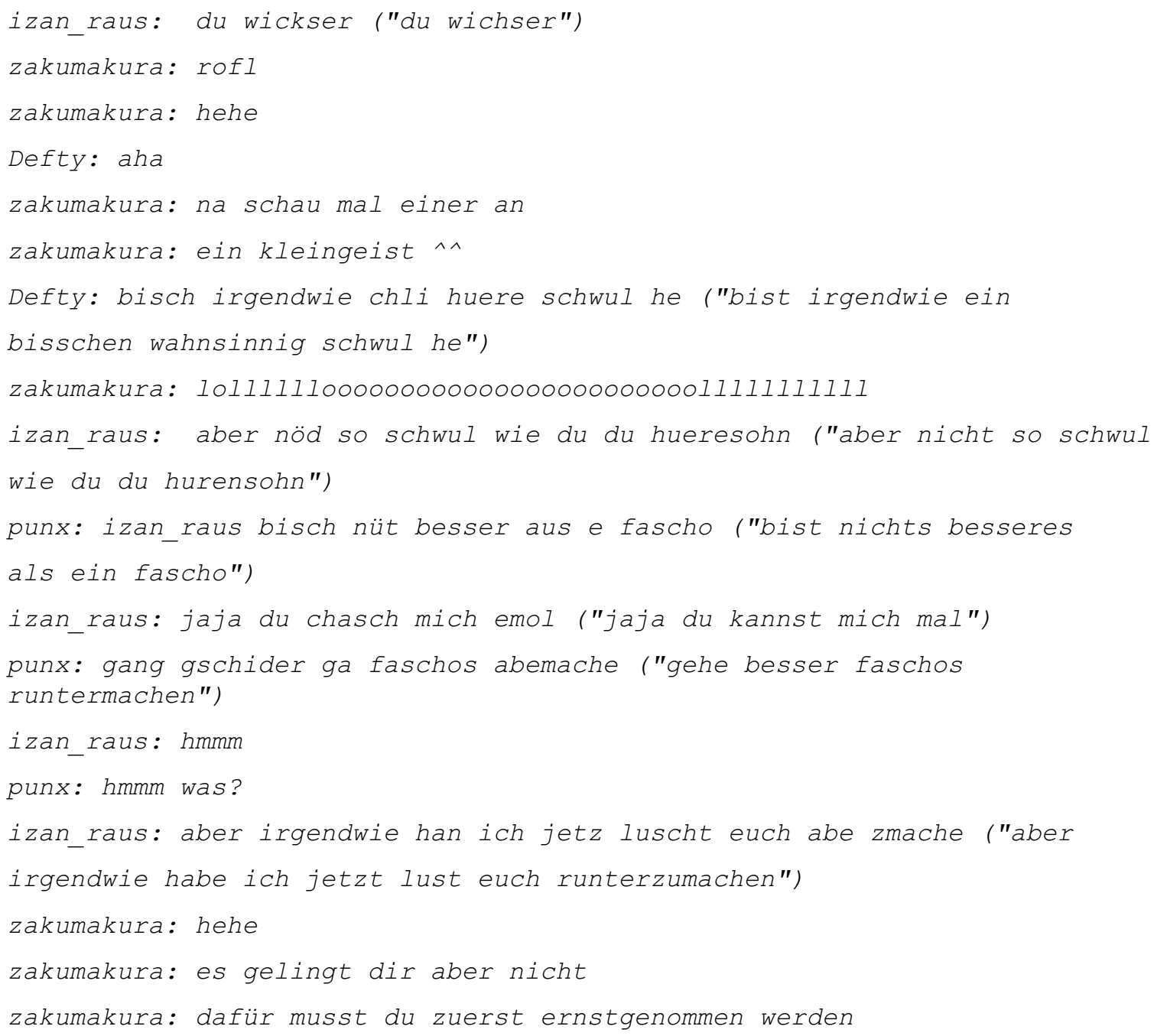

Die Veranlassung für diese Sequenz ist sicherlich das flooden von "izan_raus", dessen Name ein Anagramm von "Nazi raus" ist. Unklar ist aber die Bedeutung seiner Äußerung. Normalerweise werden Sternchen für Wörter aus dem Bereich des Genital- und Fäkalwortschatzes eingesetzt, was hier aber keinen Sinn ergibt. Allerdings verbirgt sich hinter dieser Äußerung für Insider vielleicht ein direkter Angriff. Sicher direkt angreifend ist dann die Äußerung von Defty: "chli äs problem he?" ("ein bisschen ein problem he?"). Im weiteren Verlauf ist sehr schön ersichtlich, wie die Interaktion sofort in derbe Beleidigungen und spöttische Bemerkungen verfällt. Interessant an diesem Beispiel ist auch, dass am Ende des abgedruckten Ausschnitts metakommunikative Äußerungen $\mathrm{zu}$ finden sind, welche das Verhalten von "izan_raus" negativ sanktionieren.

Im folgenden Beispiel besteht die Interaktion ebenfalls rasch darin, sich gegenseitig zu beleidigen und aufzufordern, den Channel zu verlassen (Channel "Eishockey" 3.10.02). Auch hier wird das beleidigende Verhalten also negativ sanktioniert (Rolf Ziegler ist der Name eines Verteidigers vom SCB, vom Schlittschuhclub Bern): 
Rolf_ziegler (javae=Nk93.135.202.62.dial.bluewin.ch) hat den Channel betreten

Rolf_ziegler: hallo SCB Fans

Rolf Ziegler: hallo

Cardinal zh (futzgauetel=0xpwppssj131-019-659-00.hispeed.ch) hat den Channel betreten

Rolf_ziegler: hallo

Cardinal zh: abig! ("abend!")

[1 Äußerung]

Rolf Ziegler: a lug da d'zürischachtle isch cho ("a schau die zürichschachtel ist gekommen")

[1 Äußerung]

Cardinal_zh: bin kein zürcher!!

Rolf_ziegler: doch

Rolf_ziegler: $z h$

Cardinal_zh: wohne nume da! ("wohne nur da!")

Rolf_ziegler: ebe en zürcher ("eben ein zürcher")

Cardinal_zh: mmh... ztumm zum schissse ("mmh.. zu dumm um zu scheißen/schießen")

Rolf_ziegler: du wohnsch z'züri bisch en zürcher ("du wohnst in zürich bist ein zürcher")

Rolf_ziegler: $z h^{7}=$ zwenig Hirni ("zh $=z u$ wenig Hirn")

Cardinal zh: wenni s'züri wohne heisst das dasi en zürcher bin?! ("wenn in zürich wohnen heißt dass ich ein zürcher bin?!")

Rolf_ziegler: ja für mich scho ("ja für mich schon")

[5 ̈̈ußerungen]

Rolf_ziegler: we hau ab

Cardinal_zh: nöö.

Rolf_ziegler: doch du zürcher

Cardinal_zh: jetzt aber..

Rolf_ziegler: hau ab zürcher

Cardinal_zh: nei jetzt hani aber angscht.. ("nein jetzt habe ich aber Angst. .")

Rolf_ziegler: je

Rolf_ziegler: zürcher hoseschisser ("zürcher hosenscheißer")

Rolf_ziegler: de chat ghört de Berner klar ("der chat gehört den Bernern klar")

\section{[3 Äußerungen]}

scb-girll: hey ziegler bes mol ruhig esch jo pinlech för d scb fans.. ("hey ziegler sei mal ruhig es ist ja peinlich für die scb fans")

7 "zh" ist die offizielle Abkürzung für den Kanton Zürich. 
Die Beobachtung, dass bei direkten Angriffen sofort heftig und mit Beleidigungen, Angriffen und Spott reagiert wird, lässt sich auf unterschiedliche Weisen erklären:

- Erstens dürfte die (mindestens partielle) Anonymität (s. dazu Gallery 2000) wie bei einer Nicht-Reaktion einen Einfluss darauf haben, dass sich Chatter/-innen von der kommunikativen Verantwortung, also in diesem Falle von der Verantwortung zur Höflichkeit, dispensiert fühlt. Die Kommunikationssituation im Chat baut insofern Hemmungen $a b$.

- Allerdings kann die Anonymität - und deshalb spreche ich hier von partieller Anonymität - durch Erfahrung modifiziert werden. So etwa, wenn ein Chatter aus dem Channel gekickt wird und dann mit demselben Nick wieder einloggt. Hier kann die rasch aufkommende Aggressivität der Chatter/-innen auch dadurch motiviert sein, dass sie den entsprechenden Chatter bereits 'kennen'. (vgl. weiter oben: "EHCBIEL, du dörfsch grad dusä bliebä!" ("EHCBIEL, du darfst gleich draußen bleiben"))

- Zweitens ist die para- und nonverbale Kommunikation, die für mündlich ausgetragene Streitsequenzen von großer Bedeutung ist und eine differenzierte Gestaltung von Äußerungen z.B. hinsichtlich ihres Angriffspotenzials zulassen, nur begrenzt emulierbar, einige dieser Möglichkeiten haben sogar eine technische Systemreaktion zur Folge (z.B. Großbuchstaben verwenden). Das Wegfallen dieser Differenzierungsmöglichkeiten könnte erklären, weshalb oft verbal sehr deutliche Angriffe realisiert werden.

Relativierend zur oben formulierten Beobachtung der sofort erfolgenden Gegenreaktion auf direkte Angriffe ist aber zu betonen, dass nicht alle Streitsequenzen in einer durchwegs ernsten Modalität realisiert werden.

\subsection{Die gemischte Interaktionsmodalität}

Die Streitsequenzen haben nicht immer den Status einer ernsten Interaktionsmodalität, sondern werden oft interaktiv als Mischform zwischen einer ernsten und einer humoristischen Interaktionsmodalität ausgehandelt. Darauf wurde im Zusammenhang mit dem Beispiel von "Benny1" bereits hingewiesen. So verlieren die beleidigenden Äußerungen an Schärfe, da sie einen anderen Status erhalten. Dies zeigt sich ansatzweise z.B. am Ende der Sequenz mit "Rolf_Ziegler" (Hervorhebungen von mir, M.L.):

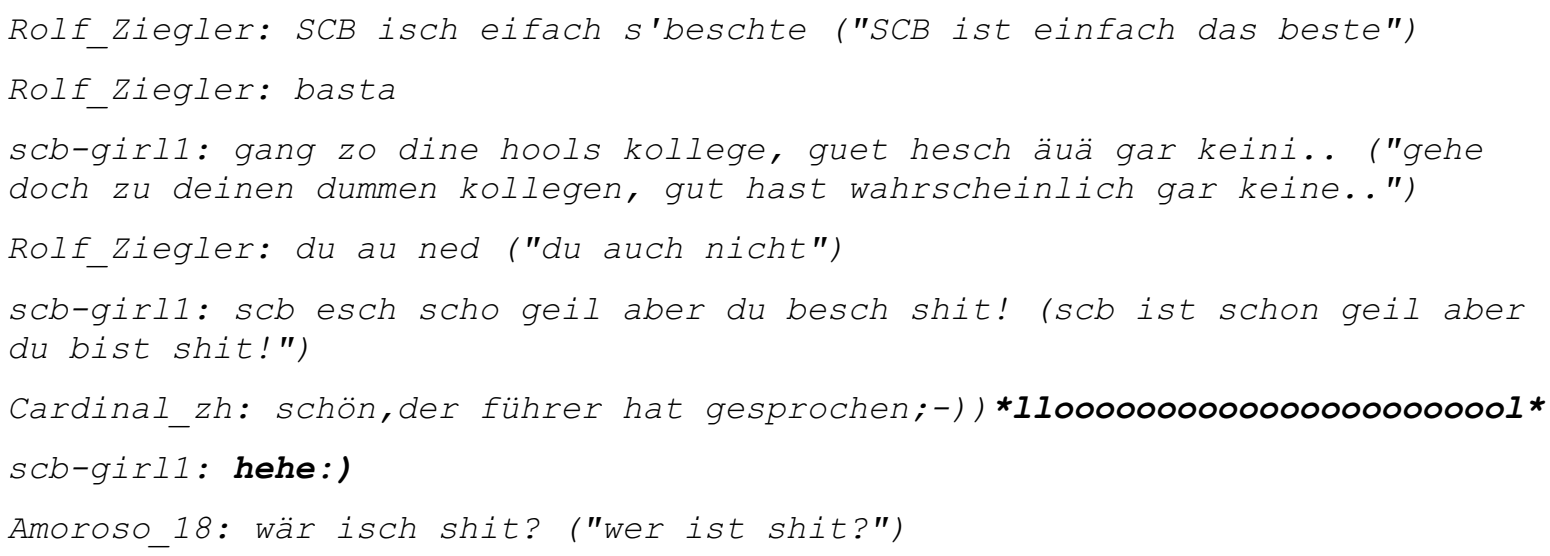




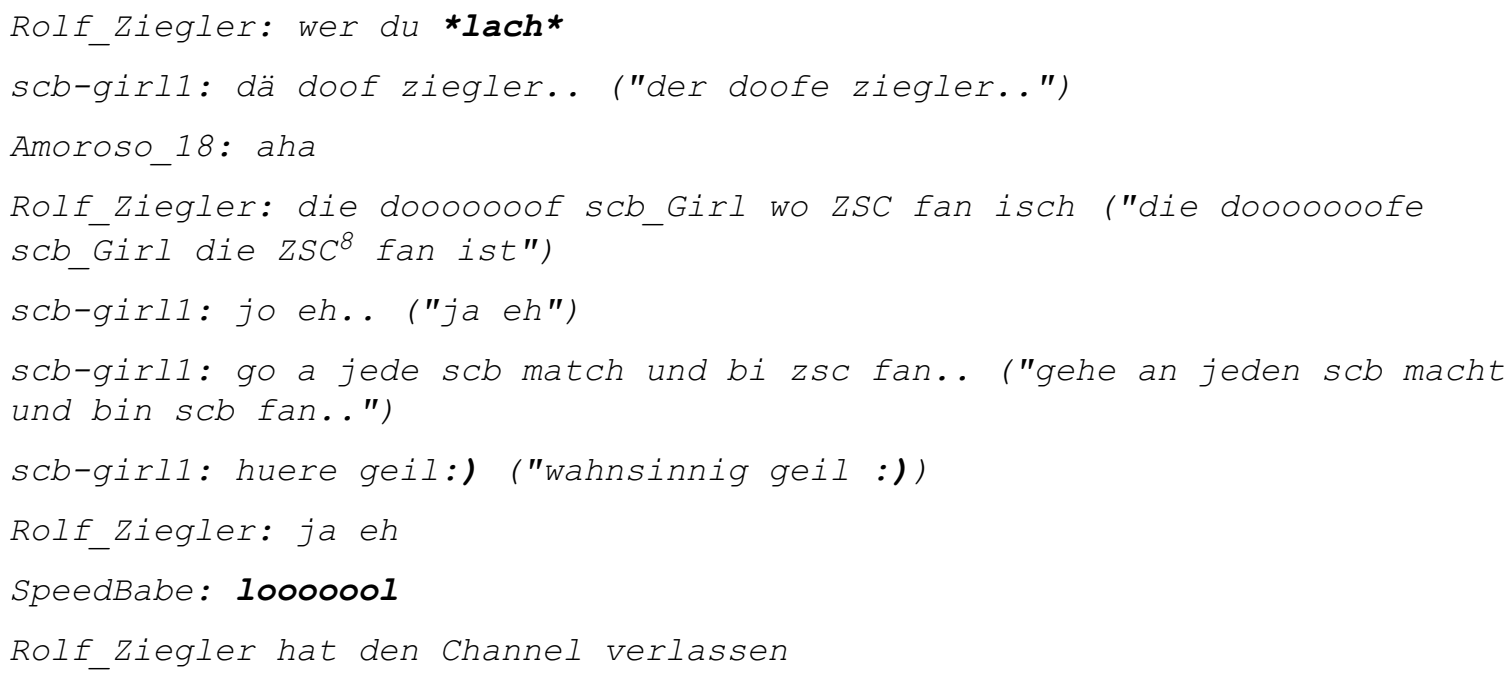

Von Anfang an in dieser gemischten Interaktionsmodalität ist die folgende Sequenz gestaltet (Channel "Eishockey" 20.10.02):

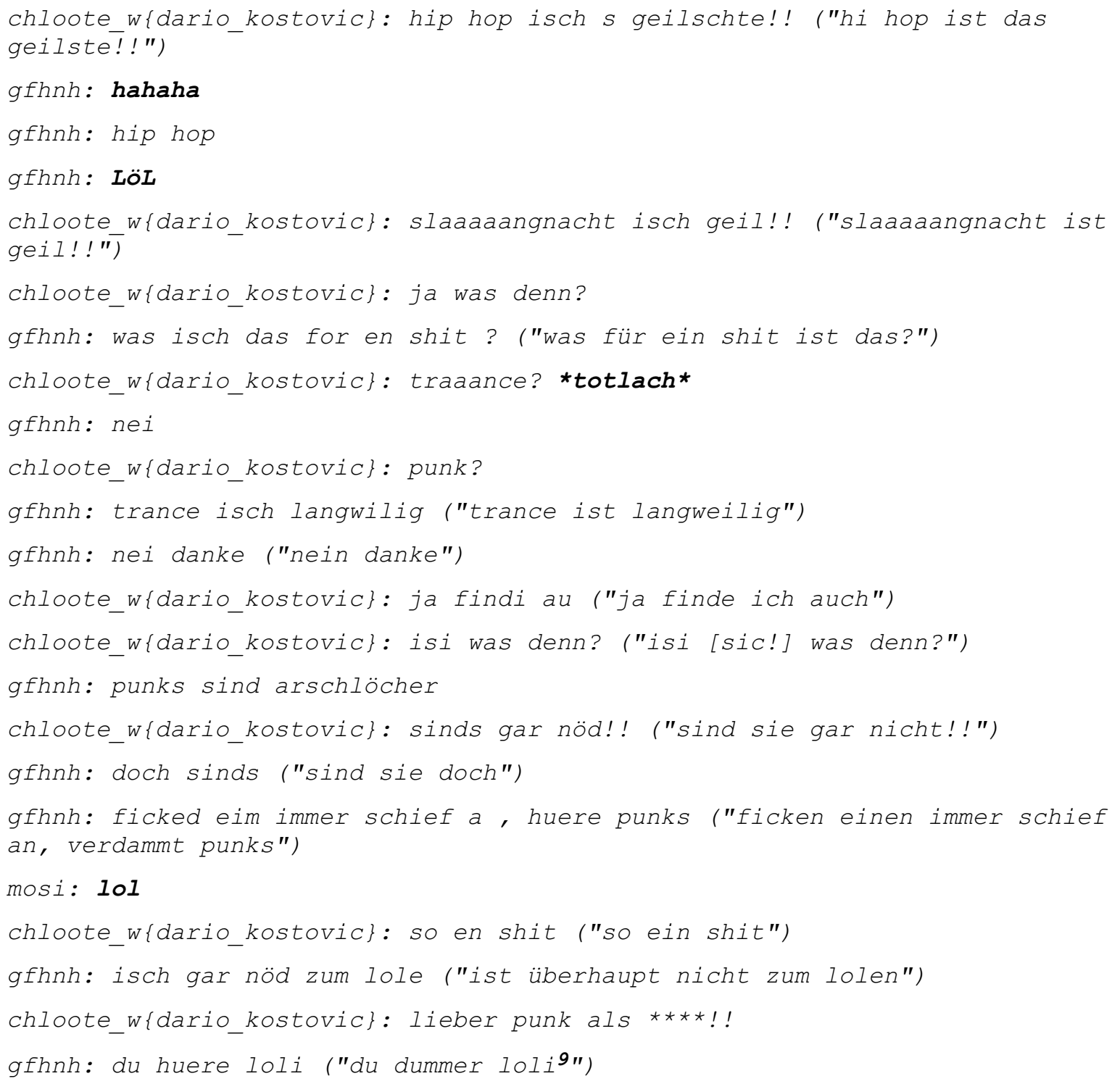

8 Zürcher Schlittschuhclub. 


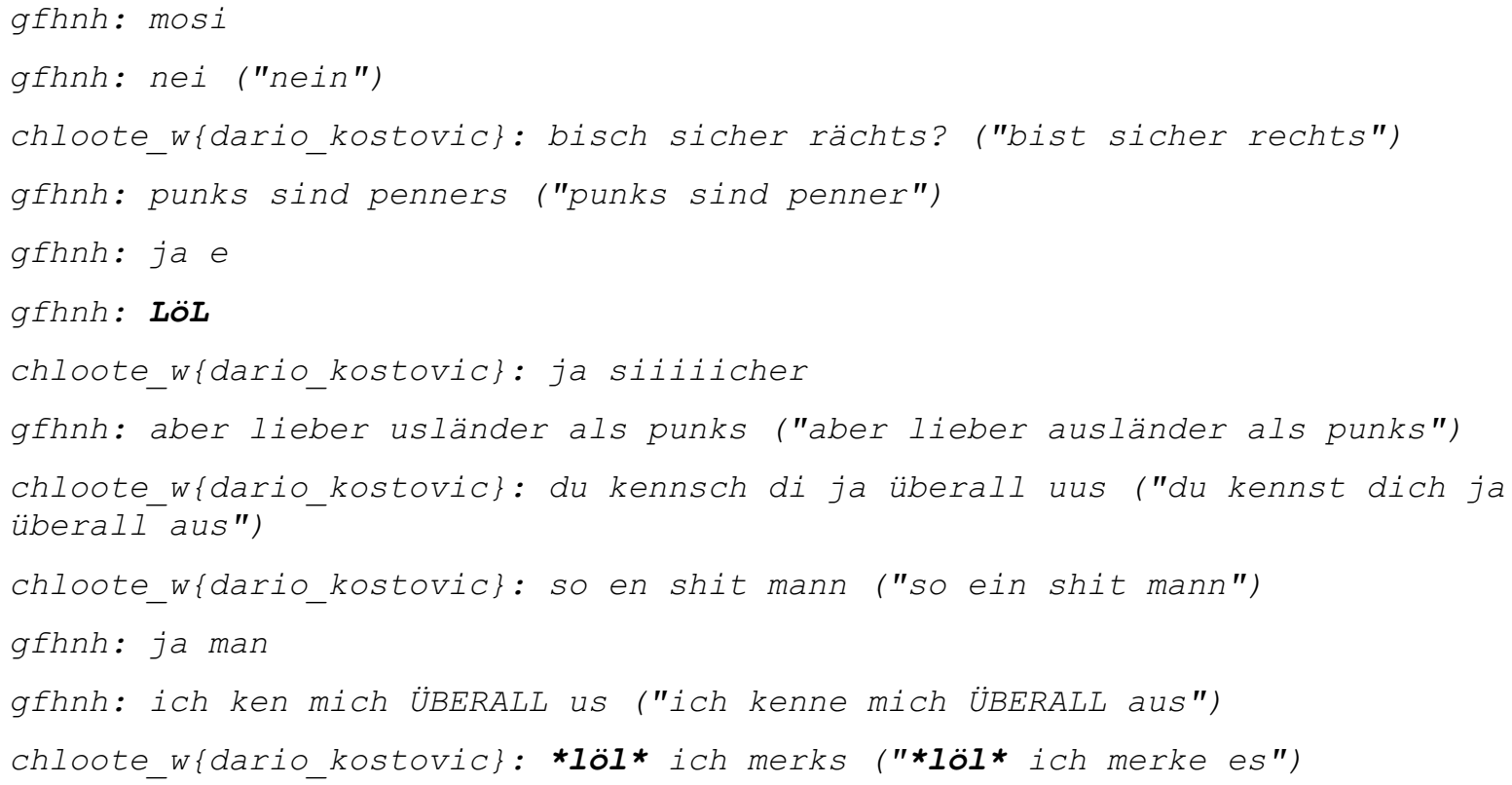

Die ganze Sequenz ist durchsetzt mit dem Akronym lol, Abwandlungen davon und verwandten Gefühlsäußerungen (etwa *totlach*), aber auch ironischen Bemerkungen ("ich ken mich ÜBERALL us" ; "ich kenne mich ÜBERALL aus") was auf eine - mindestens teilweise - scherzhafte Kommunikation hinweist. Hier rücken Streitsequenzen in die Nähe der sonstigen Kommunikation in den Chats, in denen die phatische Funktion im Vordergrund steht. Der Streit scheint hier die Funktion eines Kommunikationsspiels zu haben, über welches man mit anderen Chatter/-innen in Kontakt treten und sich profilieren kann.

\subsection{Soziale Sanktionen}

Fehlt eine humoristische Ebene, so haben die Chatter/-innen, welche einen Streit initiieren, oft negative soziale Sanktionen zu gewärtigen, wie sich in einigen Beispielen bereits gezeigt hat. Dabei sind unterschiedliche Formen von Sanktionen zu beobachten.

Im selben Protokoll vom "Eishockey"-Channel mit "Rolf_Ziegler" zum Beispiel loggt sich später ein Chatter unter "STONEY" ein. Dieser ist bei einigen Chattern als Störefried bekannt, was kurz nach dessen Einloggen thematisiert wird. Der Operator ("Noise") wird aufgefordert, sich um den Störefried zu kümmern.

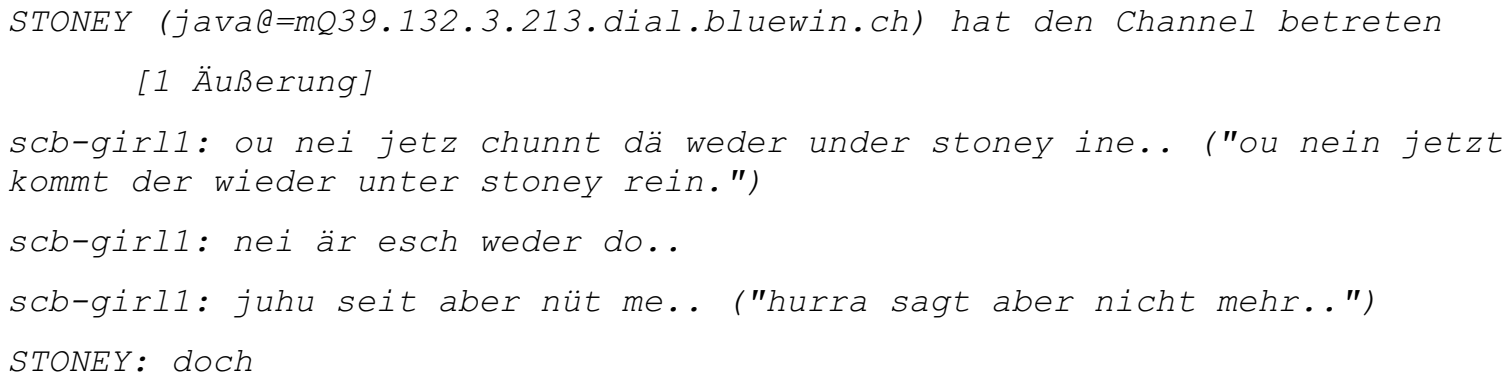

9 "loli" ist hier wahrscheinlich von "lol" abgeleitet; gleichzeitig besteht eine große Nähe zum Wort "Löli", welches im Schweizdeutschen "Dummkopf" bedeutet. 


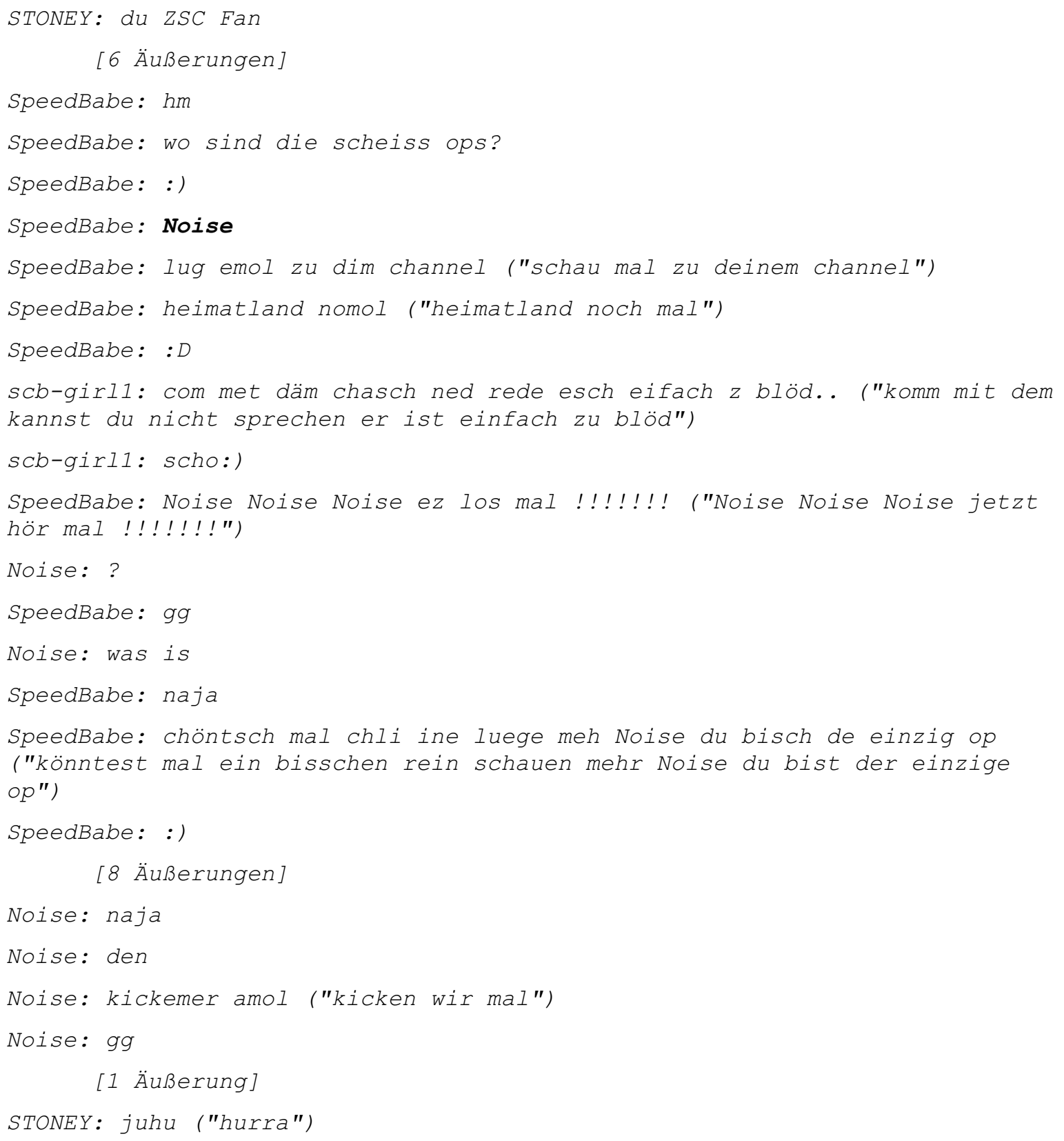

STONEY, der es offensichtlich darauf angelegt hat, herausgeworfen zu werden, scheint erfreut, wird aber nicht gekickt. Er meldet sich dann lange nicht mehr öffentlich, bis ein Chatter unter dem Nick "Forrest" einloggt:

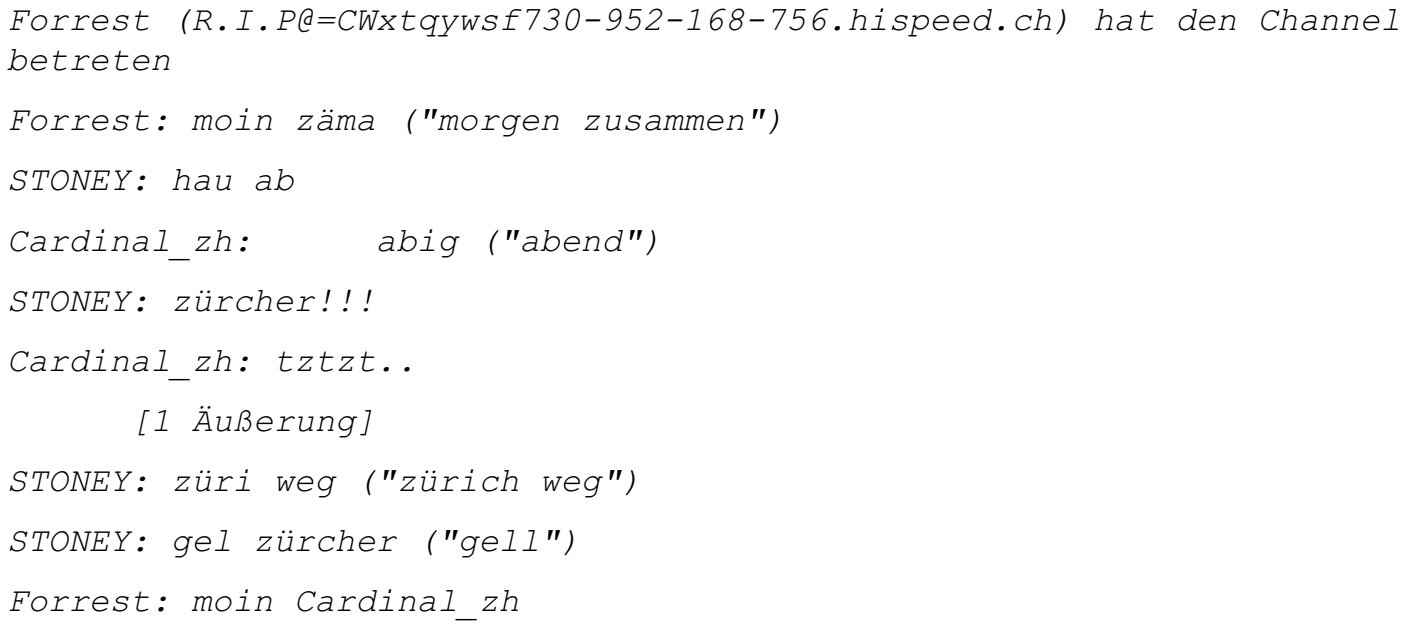


[2 Äußerungen]

Cardinal_zh: STONEY Geh doch heim, wenn du keine Freunde hast!

STONEY: fresse

Cardinal_zh: STONEY Wenn ich Deine Fresse hätte würde ich lachend in ne Kreissäge laufen!.

STONEY: haha

ChanServ hat Noise geoppt

STONEY: mich verjagts vor lache ("ich platze vor Lachen")

STONEY: hey zürcher

STONEY: $z h=$ zwenig Hirni ("zh = zu wenig Hirn")

STONEY wurde aus dem Channel von Noise geworfen $\left(\ldots:: !_{i} !\right.$ du nervst $!_{i}$ ! $: \ldots)$ ( ..: : $i$ ! SuperScript |1.0| !i!::..)

Cardinal_zh: äntli ("endlich")

STONEY (java@=fL73.132.3.213.dial.bluewin.ch) hat den Channel betreten

[3 Äußerungen]

STONEY: hihi si hei mi kickt die kinder ("hihi sie habe mich gekickt die kinder")

[2 Äußerungen]

STONEY: hey NOISE hesch spass am kicke ("hey NOISE hast du spass am kicken")

STONEY: ?

STONEY wurde aus dem Channel von Noise geworfen ( . : : ! ;! ja ! $;$ ! : :.) ( ..: :! $i$ ! SuperScript $\left.|1.0| !_{i} !:: \ldots\right)$

Lunika: Iol

STONEY (java@=Br33.132.3.213.dial.bluewin.ch) hat den Channel betreten [2 Äußerungen]

STONEY: i gloube das heisst ja ("ich glaube das heißt ja")

NOISE:STONEY: ja

[1 Äußerung]

Cardinal_zh hat IRC beendet (QUIT: User exited)

[6 Äußerungen]

ChanServ hat Noise entoppt

STONEY: hey Noise

Noise: ?

STONEY: ******* HCD

Noise: krazz

STONEY: i weiss ("ich weiß")

STONEY: kick mich doch

STONEY: kom eh wieder ("komme eh wieder")

[2 Äußerungen]

STONEY: kick mich!!!!!!!!!!!!!!!!!!!!!

SpeedBabe: den darfst wirklich

SpeedBabe: Iöl

ISSN 1615-3014 


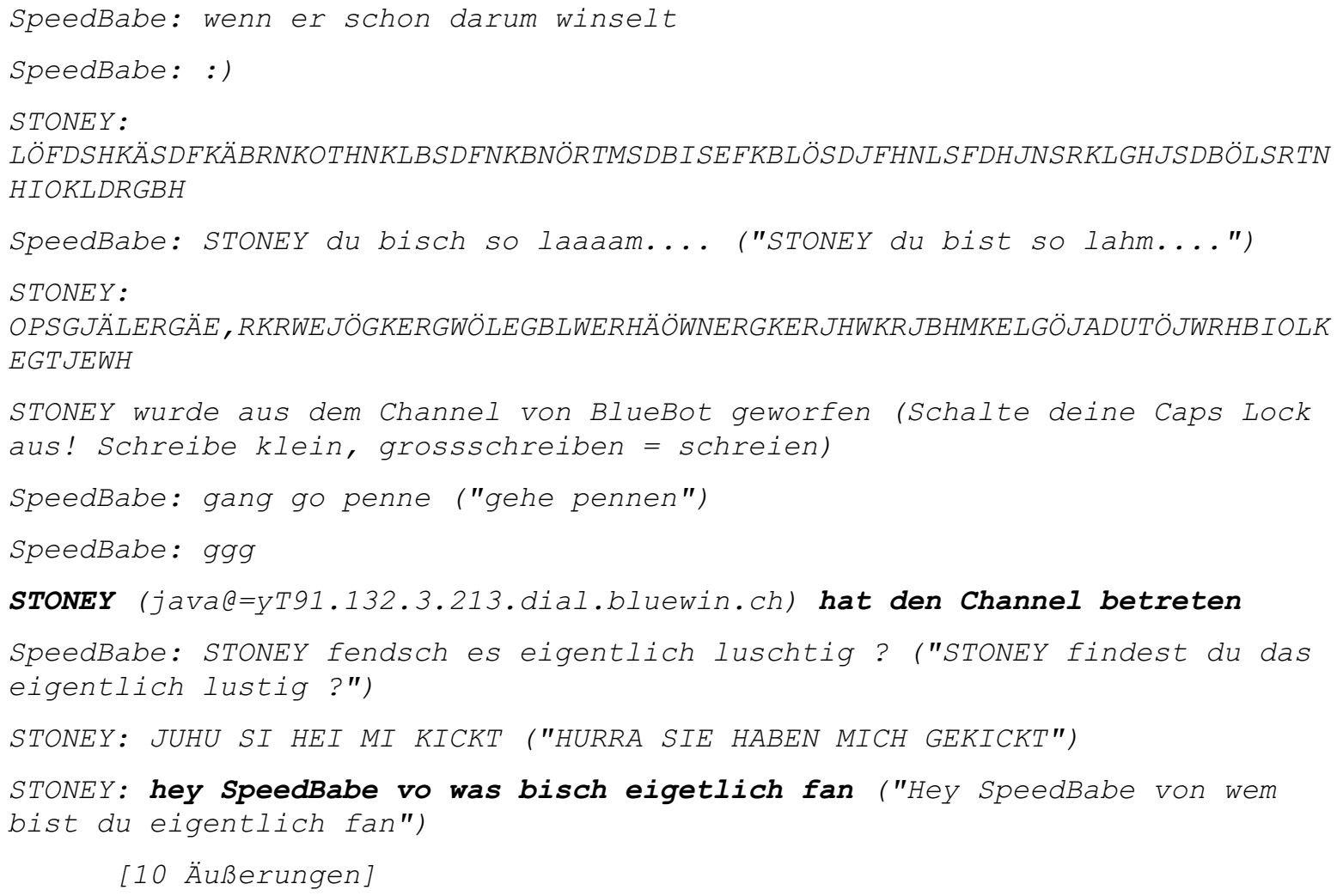

"STONEY" wird nach wenigen Äußerungen von einem Chatter aufgefordert, den Channel zu verlassen ("Geh doch heim") und wird nach weiteren Beleidigungen vom Operator "Noise" aus dem Kanal gekickt, was vom bleidigten "Cardinal_zh" mit einer positiven Sanktion kommentiert wird ("äntli", "endlich"). "STONEY" aber loggt sich sofort wieder ein und wird nach drei Äußerungen wieder vom Operator gekickt. Er loggt sich wieder ein, provoziert die Fans des Hockeyclubs Davos (HCD) und bittet darum, gekickt zu werden, was von einer Chatterin unterstuitzt wird (negative soziale Sanktion). Wie dies keine Wirkung zeigt, tippt er sinnlose Buchstabenfolgen ein, worauf er vom System aus dem Kanal gekickt wird. Wieder loggt er sich ein, wird jetzt aber - zumindest öffentlich - von allen ignoriert, worauf er sich selbst ausloggt. Interessant hier ist nicht nur die Tatsache, dass sein Verhalten negativ sanktioniert wird, sondern auch, dass er Äußerungen produziert, die in der mündlichen Kommunikation kein Pendant haben, die also nicht konzeptionell mündlich sind.

Ähnliches liegt in folgender Sequenz vor (Channel "Punk", 3.10.02):

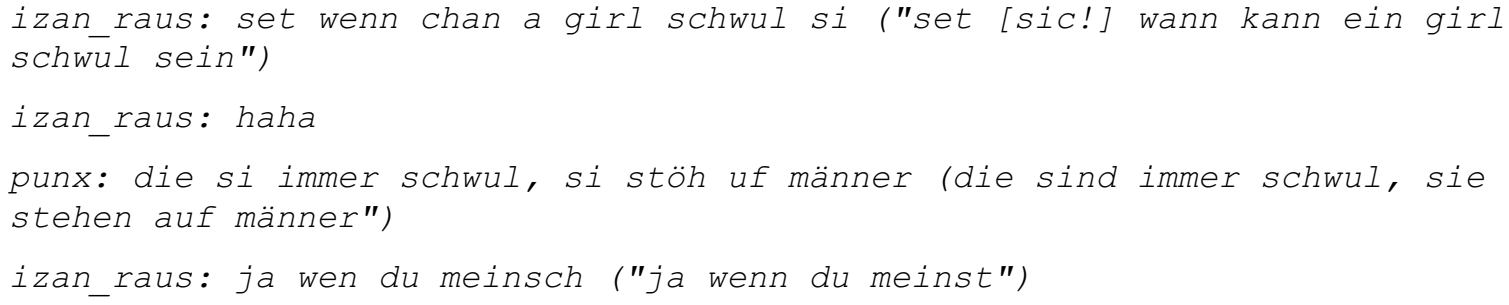




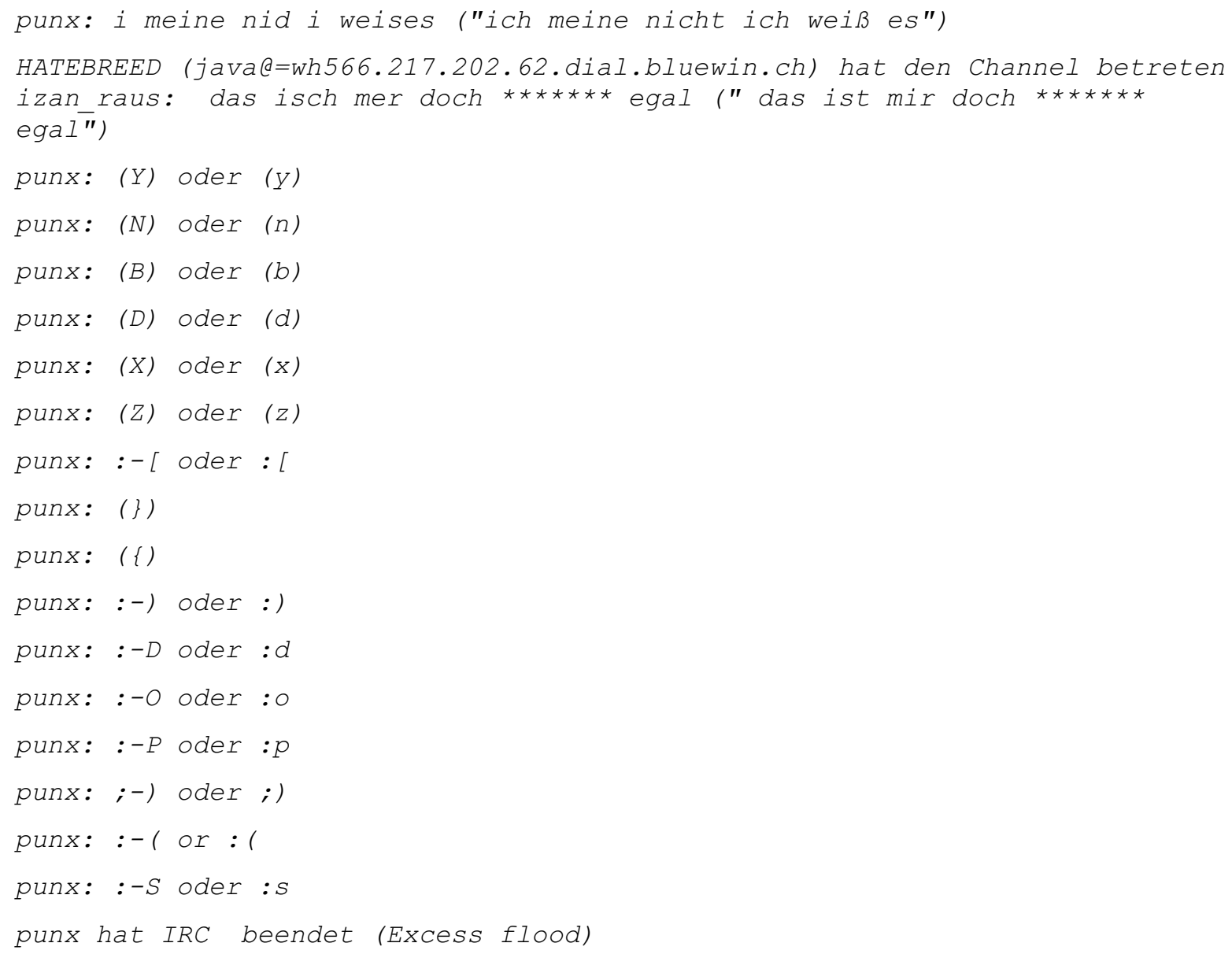

Neben dem sozialen Channel-Verweis (also der Aufforderung abzuhauen) und der Aufforderung an den Operator, etwas gegen einen Chatter zu unternehmen, finden sich in meinem Korpus folgende Sanktionen:

- Aufforderung, still zu sein: "benny, häb dini sch**** F R à S S E!!!" ("benny, halte deine sch**** F R E S S E!!!")

scb-girll: hey ziegler bes mol ruhig esch jo pinlech för d scb fans.. ("hey ziegler sei mal ruhig es ist ja peinlich für die scb fans")

- Negative Bewertung des Kommunikationsverhaltens: leamas: u du ufem aafiggitrip? ("und du auf dem anficktrip")

- Entsolidarisierung: EVZ_4_ever: hei evz-fan gang di nick name go ändere das esch jo en schand (hei evz-fan gehe deinen nick name ändern das ist ja eine schande")

- Verweigerung, jemanden als Kommunikationspartner anzuerkennen: zakumakura: dafür musst du zuerst ernstgenommen werden

- Beschimpfungen: "bisch nüt besser aus e fascho" ("bist nichts besseres als ein fascho")

- Spott: schoggi-tiger: ou du machsch mer angscht ("ou du machst mir aber angst")

- Verweis auf Chatiquette: jasinta: es giltet do es verbot übrigens geg alli rassischte,antisemitischtem,...... ("es gilt hier ein verbot übrigens gegen alle rassistischen, anitsemitischen,......") 
Die Analyse ergab, dass in den meisten Streitsequenzen soziale, negative Sanktionen von anderen Chattern zu finden sind, welche an denjenigen Chatter gerichtet sind, der als Veranlasser des Streits identifiziert wird. Die meisten dieser Sanktionen sind, wie die Beispiele zeigen, so formuliert, dass der angesprochene Chatter direkt negativ bewertet wird. Sie tragen deshalb meist nichts zu einer Deeskalation bei.

\subsection{Streitbeendigung}

Die meisten Streitsequenzen, die im vorliegenden Korpus zu finden sind, werden ganz abrupt beendet. Entweder, weil ein Beteiligter durch eine technische Sanktion aus dem Channel gekickt wird, oder weil der Streit nicht weiter ausgetragen wird - mindestens nicht öffentlich. Die Tatsache, dass in den meisten Belegen mindestens einer der Beteiligten nach Abbruch der Streitsequenz sofort weiterhin rege am Chat teilnimmt, weist jedoch darauf hin, dass die Kommunikation abgebrochen und nicht privat weitergeführt wurde. Ein derartiger Abbruch, der in der face-to-face-Kommunikation schwer beziehungsbelastend wäre, scheint im Chat üblich zu sein.

Diese Beobachtung deckt sich mit anderen Analyseergebnissen (z.B. Schönfeldt 2001: 4849), dass im Chat - bis auf Verabschiedungssequenzen - Gesprächsbeendigungen oft fehlen. Meist wenden sich die Chatter/-innen einfach anderen Chattern zu.

\section{$5 \quad$ Fazit}

In den hier untersuchten Chats wird selten gestritten. Wenn gestritten wird, dann wird dies meist veranlasst durch direkte Angriffe und Beleidigungen, und die daran anschließenden Streitsequenzen zeichnen sich dadurch aus, dass rasch die Beziehungsebene in den Vordergrund rückt und Äußerungen realisiert werden, welche den Kommunikationspartner direkt bewerten, und zwar sehr negativ. Hinzu kommen als Ausdruck von Emotionalität die Reduplikation von Satzzeichen (schoggi-tiger: benny hou ab!!!!!!! "benny hau ab!!!!!!") oder von Buchstaben (Rolf_Ziegler: die dooooooof scb_Girl wo ZSC fan isch Übersetzung? (" "die dooooooofe scb_Girl die ZSC fan ist").Zum Teil sind diese Sequenzen auch mit Äußerungen durchsetzt, die markieren, dass die Beleidigungen nicht ganz ernst gemeint sind, wodurch eine Mischung verschiedener Interaktionsmodalitäten entsteht. Fehlen derartige Äußerungen, welche die Ernsthaftigkeit der Angriffe relativieren, so wird das Verhalten des Streitveranlassers meist negativ sanktioniert. Man könnte dies als Form sozialer Kontrolle interpretieren. Diese Sanktionen beinhalten aber meist wiederum negativ bewertende Äußerungen, sodass sie kaum zu einer Deeskalation beitragen.

Viele Streitsequenzen werden nicht beendet, sondern einfach abgebrochen. Wenn eine Deeskalation zu beobachten ist, so meist in Form von einer Wendung ins Humoristische.

Streitsequenzen finden sich vor allem in kleineren Chat-Channels.

Die hier analysierten Streitsequenzen zeichnen sich dadurch aus, dass nie unterschiedliche Standpunkte ausgehandelt werden und so die zu Grunde liegenden Konflikte nicht gelöst 
werden. Das Lösen von Konflikten kann also nicht die Funktion dieser Sequenzen sein. Viel mehr spielen wahrscheinlich die Funktionen der Provokation und der Profilierung eine Rolle.

Da im Chat anonymes Kommunizieren möglich ist, kann man die Wirkung des eigenen Verhaltens auf andere eher vernachlässigen und sich ungehemmter auf die eigenen Bedürfnisse konzentrieren. Man kann deshalb seinen Aggressionen ungebremst Ausdruck verleihen und andere provozieren (dazu auch Döring 2001: 112). Eine Provokation ist deshalb im Chat gut möglich, da die Kommunikation in diesem Medium auch Nähe vermittelt (Storrer 2001a). Diese Kombination von anonymer Kommunikation bei gleichzeitiger Übermittlung von Nähe ist ein zentrales Merkmal der elektornischen Schriftlichkeit, wie sie im Chat zu finden ist (Thimm 2001: 260) ${ }^{10}$

In gewissen Streitsequenzen, die eine Wendung ins Humoristische nehmen oder aber von Anfang an in einer gemischten Interaktionsmodalität realisiert werden, ist es möglich, sich als scharfzüngigen, schlagfertigen, aber dennoch witzigen Chatter zu profilieren. Hier dürfte wie in einem großen Teil der sonstigen Chat-Kommunikation - die phatische Funktion überwiegen.

Streitsequenzen im Chat weisen so nicht nur Unterschiede im Hinblick auf die formalen Merkmale von Streitsequenzen in der face-to-face-Kommunikation auf (rasche Eskalation, keine Aushandlungsphase, oft fehlende Beendigung etc.), sondern auch im Hinblick auf die Funktion. Es bleibt deshalb zu diskutieren, ob sie sinnvollerweise als "schriftlich realisierte Gespräche" (Schönfeldt 2001: 52) bezeichnet werden sollen. Problematisch machen eine solche Bezeichnung auch diese Äußerungen, die nicht konzeptionell mündlich sind (Großbuchstaben ohne Sinn).

Genauer zu untersuchen bleiben kulturelle Vergleiche einzelner Streitsequenzen, wobei hier nicht nur an Chats aus anderen Ländern zu denken ist, sondern auch an einen Vergleich unterschiedlicher Gruppenkulturen - Hinweise auf derartige Unterschiede zeigten sich schon im hier untersuchten Korpus. Damit verbunden ist die Frage nach der Funktion sozialer negativer Sanktionen im Hinblick auf eine Gruppenbildung in einzelnen Channels. Ebenfalls wichtig wäre eine Ergänzung durch Analysen von Chats, in denen nicht in erster Linie geplaudert wird, sondern ernsthaft diskutiert, zum Beispiel über politische Themen. Und nicht zuletzt müssten auch Flirt-Chats auf Streitsequenzen hin untersucht werden - gehören sie doch nach wie vor zu den am häufigsten frequentieren Channels.

\section{Literaturangaben}

Döring, Nicola (2001): "Belohnungen und Bestrafungen im Netz: Verhaltenskontrolle in Chat-Foren". Gruppendynamik und Organisationsberatung 2: 109-143.

Gallery, Heike (2000): "'bin ich-klick ich' - Variable Anonymität im Chat". In: Thimm, Caja (ed.): Soziales im Netz. Sprache, Beziehungen und Kommunikationskulturen im Internet. Opladen: 71-88.

\footnotetext{
${ }^{10}$ Eine Entsprechung - allerdings unter umgekehrten Vorzeichen - finden diese Provokationen in besonders prosozialer Kommunikation im Chat, dazu Döring (2001: 111).
} 
Gruber, Helmut (1996): Streitgespräche. Zur Pragmatik einer Diskursform. Opladen.

Haase, Martin et al. (1997): "Internetkommunikation und Sprachwandel". In: Weingarten, Rüdiger (ed.): Sprachwandel durch Computer. Opladen: 51-85.

Sassen, Claudia (2000): "Phatische Variabilität bei der Initiierung von Internet-Relay-ChatDialogen". In: Thimm, Caja (ed.): Soziales im Netz. Sprache, Beziehungen und Kommunikationskulturen im Internet. Opladen: 89-108.

Schönfeldt, Juliane (2001): "Die Gesprächsorganisation in der Chat-Kommunikation". In: Beißwenger, Michael (ed.): Chat-Kommunikation. Sprache, Interaktion, Sozialität \& Identität in synchroner computervermittelter Kommunikation. Perspektiven auf ein interdisziplinäres Forschungsfeld. Stuttgart: 25-53.

Spiegel, Carmen (1995): Streit. Eine linguistische Untersuchung verbaler Interaktionen in alltäglichen Zusammenhängen. Tübingen. (= Forschungsberichte des Instituts für deutsche Sprache 75)

Storrer, Angelika (2001a): "Getippte Gespräche oder dialogische Texte? Zur kommunikationstheoretischen Einordnung der Chat-Kommunikation". In: Lehr, Andrea et al. (eds.): Sprache im Alltag. Beiträge zu neuen Perspektiven in der Linguistik. Herbert Ernst Wiegand zum 65. Geburtstag gewidmet. Berlin etc.: 439-465.

Storrer, Angelika (2001b): "Sprachliche Besonderheiten getippter Gespräche: Sprecherwechsel und sprachliches Zeigen in der Chat-Kommunikation". In: Beißwenger, Michael (ed.): Chat-Kommunikation. Sprache, Interaktion, Sozialität und Identität in synchroner, computervermittelter Kommunikation. Perspektiven auf ein interdisziplinäres Forschungsfeld. Stuttgart: 3 - 24.

Thimm, Caja (2001): "Funktionale Stilistik in elektronischer Schriftlichkeit: Der Chat als Beratungsforum". In: Beißwenger, Michael (ed.): Chat-Kommunikation. Sprache, Interaktion, Sozialität \& Identität in synchroner computervermittelter Kommunikation. Perspektiven auf ein interdisziplinäres Forschungsfeld. Stuttgart: 255-278.

Wichter, Sigurd (1991): Zur Computerwortschatz-Ausbreitung in der Gemeinsprache. Elemente der vertikalen Sprachgeschichte einer Sache. Frankfurt am Main. 